\title{
Magnetic Nanoparticles: The Simulation of Thermodynamic Properties
}

\author{
U. Nowak and D. Hinzke
}

Theoretische Physik, Gerhard-Mercator-Universität, 47048 Duisburg, Germany

\begin{abstract}
Magnetic materials are now controllable down to a nanometer length scale and, hence, there is a broad interest in the understanding of magnetic phenomena in nanostructured systems. With decreasing size thermal activation becomes more and more relevant and the understanding of the role of temperature for the dynamic behavior and for the magnetic stability of ferromagnetic nanostructures is an important subject in micromagnetism. It is interesting from a fundamental point of view as well as for applications in magnetic devices.

In this article, an overview is given on numerical approaches for the investigation of thermodynamic properties of magnetic systems, described by classical spin systems. As an example, magnetization reversal in nanostructures is simulated over a wide range of time-scales, from fast switching processes on a picosecond time-scale to thermally activated reversal on a microsecond time-scale. Langevin dynamics is used as well as a time quantified Monte Carlo method for the simulation of elongated $\mathrm{Co}$ nanoparticles. We study the behavior of the magnetization during the reversal, the energy barriers which are relevant for the thermally activated longtime behavior and the corresponding characteristic times.
\end{abstract}

\section{Introduction}

The miniaturization of magnetic structures plays an important role for fundamental research as well as for technical applications, e. g. in magnetic storage devices and sensors [1]. New experimental techniques allow for a preparation and investigation of magnetic systems of smaller and smaller spatial extension [2]. This leads to an incremental interest in the understanding of the behavior of small magnetic particles and structures down to the nanometer scale. Patterned media consisting of magnetic particles which are small enough to be in a single-domain remanent state are expected to enhance the storage density. But with decreasing size of the magnetic system thermal activation becomes relevant. Hence, much effort is focused now on the understanding of magnetization dynamics at finite temperatures since thermal activation reduces the stability of the magnetic state and sets the ultimate limit for the density of data storage, the so-called superparamagnetic limit [3].

In the following, we describe numerical techniques for the study of magnetic nanostructures modeled as classical spin systems, taking into account finite temperatures. In the next chapter, we will focus on the underlying model 
and the two basic methods, Langevin dynamics [4] and Monte Carlo methods [5]. In Chapter 3 , as an example, magnetization reversal in Co nanoparticles is considered. Starting with the deterministic spin dynamics on short time scales which plays a crucial role in high-speed data storage $[6,7,8]$, we go on to the probabilistic long-time behavior where a thermally assisted reversal can occur even for magnetic fields below the coercive field $[9,10,11,12,13]$.

\section{Model and Simulations}

In this section we will describe the underlying model as well as the basic methods for the simulation of thermodynamic properties in magnetic systems.

\subsection{Spin Model versus Continuum Theory}

The micromagnetic properties of a system can be described using a model of classical magnetic moments which are localized on a given lattice. Such a spin model can be motivated following different lines: on the one hand it is the classical limit of a quantum mechanical, localized spin model the Heisenberg model [14]. On the other hand, a classical spin model can also be interpreted as the discretized version of a micromagnetic continuum model [15], where the charge distribution for a single cell of the discretized lattice is approximated by a point dipole $[14,16]$.

The interpretation as an atomic model restricts the use of computer simulations to the investigation of rather small systems of only a few million atoms - corresponding to particle sizes of only a few nanometers. On the other hand, within a continuum model, the space might be discretized on a much larger length scale, as compared to an atomic distance. However, in continuum theory usually a constant absolute value of the magnetization vector is assumed, an assumption which fails for higher temperatures since the space averaged magnetization breaks down when approaching the critical temperature. Hence, one can expect to get correct thermal properties only in the limit of small cell sizes of the order of atomic distances.

In the following, let us consider a classical three dimensional Heisenberg Hamiltonian for localized spins,

$$
\begin{aligned}
\mathcal{H}= & -J \sum_{\langle i j\rangle} \mathbf{S}_{i} \cdot \mathbf{S}_{j}-\mu_{s} \mathbf{B} \cdot \sum_{i} \mathbf{S}_{i}-d_{z} \sum_{i}\left(S_{i}^{z}\right)^{2} \\
& -w \sum_{i<j} \frac{3\left(\mathbf{S}_{i} \cdot \mathbf{e}_{i j}\right)\left(\mathbf{e}_{i j} \cdot \mathbf{S}_{j}\right)-\mathbf{S}_{i} \cdot \mathbf{S}_{j}}{r_{i j}^{3}}
\end{aligned}
$$

where the $\mathbf{S}_{i}=\mu_{i} / \mu_{s}$ are three dimensional magnetic moments of unit length representing atomic magnetic moments in a point dipole approximation. The first sum is the ferromagnetic exchange of the moments with the coupling constant $J$. The second sum is the coupling of the magnetic moments to an 
external magnetic field $B$, the third sum represents a uniaxial anisotropy, here, for $d_{z}>0$, favoring the $z$ axis as easy axis of the system, and the last sum is the dipolar interaction where $w=\mu_{0} \mu_{s}^{2} /\left(4 \pi a^{3}\right)$ describes the strength of the dipole-dipole interaction. The $\mathbf{e}_{i j}$ are unit vectors pointing from lattice site $i$ to $j$ and $r_{i j}$ is the distance between these lattice sites in units of $a$.

The transformation of the above introduced atomic parameters to the material parameters which are usually used in a continuum model is given by $J=2 a A_{x}$ where $A_{x}$ is the exchange energy, $d_{z}=K a^{3}$ where $K$ is the anisotropy energy density and $\mu_{s}=M_{s} a^{3}$ where $M_{s}$ is the spontaneous magnetization.

A fundamental physical understanding of thermally activated magnetization dynamics requires studies over the whole time range from a picosecond to the so-called 'slow dynamic' behavior arising from thermally excited decay of metastable states over time scales from nanoseconds upwards. In the short time limit spin precession is important which can be taken care of by studying the corresponding equation of motion. In the long time limit only spin relaxation and thermal fluctuations are relevant which can be studied very conveniently using Monte Carlo methods with quantified time step [5]. In both cases, the dipole-dipole interaction can be computed efficiently with the help of fast Fourier transformation (FFT) methods $[17,18]$. One should however note that in a Monte Carlo simulation with a single-spin flip algorithm the FFT method is an approximation the implementation of which was described in details before [13].

\subsection{Landau-Lifshitz-Gilbert Equation with Langevin Dynamics}

The basic numerical approach for the description of spin dynamics including thermal activation is the direct numerical integration of the Langevin equation of the problem. In order to obtain thermal averages one has to calculate many of these trajectories starting with the same initial conditions, taking an average over these trajectories for the quantities of interest. This method is referred to as the Langevin dynamics formalism [4].

The underlying equation of motion for a magnetic system is the LandauLifshitz-Gilbert (LLG) equation,

$$
\frac{\partial \mathbf{S}_{i}}{\partial t}=-\frac{\gamma}{\left(1+\alpha^{2}\right) \mu_{s}} \mathbf{S}_{i} \times\left[\mathbf{H}_{i}(t)+\alpha\left(\mathbf{S}_{i} \times \mathbf{H}_{i}(t)\right)\right]
$$

with the gyromagnetic ratio $\gamma=1.76 \times 10^{11}(\mathrm{Ts})^{-1}$, the dimensionless damping constant $\alpha$, and the internal field $\mathbf{H}_{i}(t)=\boldsymbol{\zeta}_{i}(t)-\partial \mathcal{H} / \partial \mathbf{S}_{i}$. Langevin dynamics is introduced here in form of the noise $\zeta_{i}(t)$ which represents thermal fluctuations, with $\left\langle\zeta_{i}(t)\right\rangle=0$ and $\left\langle\zeta_{i}^{\eta}(t) \zeta_{j}^{\theta}\left(t^{\prime}\right)\right\rangle=2 \delta_{i j} \delta_{\eta \theta} \delta\left(t-t^{\prime}\right) \alpha k_{\mathrm{B}} T \mu_{s} / \gamma$ where $i, j$ denote once again lattice sites and $\eta, \theta$ Cartesian components.

The LLG equation with Langevin dynamics is a stochastic differential equation with multiplicative noise. For this kind of differential equation a 
problem arises which is called the Itô-Stratonovich dilemma [19]. As a consequence, different time discretization schemes may converge to different results with decreasing time step. As was pointed out in [20] the multiplicative noise in the Langevin equation above has to be treated by means of the Stratonovich interpretation. Hence, in order to obtain useful numerical results one has to use adequate methods. The simplest appropriate discretization scheme leading to a Stratonovich interpretation is the Heun method $[19,20]$ which is used in the following.

\subsection{Monte Carlo Methods}

Within a Monte Carlo approach [21] trajectories in phase space are calculated following a master equation [22] for the time development of the probability distribution $P_{s}(t)$ in phase space,

$$
\frac{\mathrm{d} P_{s}}{\mathrm{~d} t}=\sum_{s^{\prime}}\left(P_{s^{\prime}} w_{s^{\prime} \rightarrow s}-P_{s} w_{s \rightarrow s^{\prime}}\right) .
$$

Here, $s$ and $s^{t}$ denote different states of the system and $w_{s^{\prime} \rightarrow s}$ is the transition rate for a change from a state $s^{\prime}$ to a state $s$. These rates have to fulfill the condition [22]

$$
\frac{w_{s \rightarrow s^{\prime}}}{w_{s^{\prime} \rightarrow s}}=\exp \left[\frac{E(S)-E\left(S^{\prime}\right)}{k_{\mathrm{B}} T}\right]
$$

The master equation describes exclusively the coupling of the system to the heat bath [22]. Hence, only the irreversible part of the dynamics of the system is considered including only the relaxation and the fluctuations. A Monte Carlo simulation does not include the energy conserving part of the equation of motion. Hence, no precession of magnetic moments will be found.

Monte Carlo approaches in general have no physical time associated with each step of the algorithm, so that an unquantified dynamic behavior is represented. However, recently a time quantified Monte Carlo method was proposed in [5] and later successfully applied to different model systems $[12,13,23]$. Here, the interpretation of a Monte Carlo step as a realistic time interval $\Delta t$ was achieved by a comparison of one step of the Monte Carlo process with a time interval of the LLG equation in the high damping limit. We will use this algorithm in the following. The trial step of this algorithm is a random movement of the magnetic moment within a cone with a given size $r$ with

$$
r^{2}=\frac{20 k_{B} T \alpha \gamma}{\left(1+\alpha^{2}\right) \mu_{s}} \Delta t
$$

In order to achieve this efficiently one constructs a random vector with constant probability distribution within a sphere of radius $r$. This random vector is added to the initial moment and subsequently the resulting vector is normalized [5]. 
Using this algorithm one Monte Carlo step represents a given time interval $\Delta t$ of the LLG equation in the high damping limit as long as $\Delta t$ is chosen appropriately (for details see [24]).

\section{Simulation of Co Nanoparticles}

As an example, in the following we consider a finite, ellipsoidal Co particle with length $L=4 \mathrm{~nm}$ and diameter $D=2 \mathrm{~nm}$. The corresponding material parameters are $A_{x}=1.3 \cdot 10^{-11} \mathrm{~J} / \mathrm{m}, K=6.8 \cdot 10^{5} \mathrm{~J} / \mathrm{m}^{2}$ and $M_{s}=1.401 \cdot 10^{6}$ $\mathrm{A} / \mathrm{m}$. For simplicity we simulate a simple cubic lattice with atomic distance $a=0.25 \mathrm{~nm}$. Our simulation starts with a spin configuration where all magnetic moments point into the $z$ direction, aligned with the easy axis, and with the $z$ component of the external magnetic field antiparallel to the magnetization so that the system is in an unstable, or at least metastable state.

In sufficiently small particles the magnetic moments rotate coherently during the magnetization reversal. A quantitative description of coherent rotation in ellipsoidal single domain particles was developed by Stoner and Wohlfarth [25]. Depending on the angle between the applied field $\mathbf{B}$ and the $z$ (easy) axis of the system, the coercive field $B_{c}$ varies following the so-called Stoner-Wohlfarth asteroid [25]. Under an angle of $45^{\circ}$ the coercive field $B_{c}$ is given by $B_{c}=d_{\mathrm{eff}} V / \mu_{\mathrm{s}}$ where $V$ is the volume of the particle and $d_{\mathrm{eff}}$ is an effective anisotropy constant. In our case it is $B_{c} \approx 0.7 \mathrm{~T}$.

We will study the magnetization reversal in two limiting cases, for large magnetic fields above the Stoner-Wohlfarth limit in the low damping limit and for smaller magnetic fields in the high damping limit, respectively.

\subsection{Precessional Reversal}

First, we are interested in fast switching processes where the applied field is higher than the coercive field and the reversal is dominated by spin precession. In this case there is no energy barrier and the reversal process starts immediately. Figure 1 shows the time dependence of the magnetization of our Co particle in the low damping limit $(\alpha=0.1)$. Here, we simulate the system with Langevin dynamics as described before.

The $z$ component of the magnetization is shown as well as the $x$ component. The magnetic field $\mathbf{B}$ is set under an angle of $45^{\circ}$ to the $z$ axis within the $y z$ plane so that the response of the system to the external field sets in directly. The wavering magnetization of the system clearly follows from the precession of the spins. Note, that the precession time of our system is not simply given by the precession time of a single spin in an external field $\left(\tau_{p}=2 \pi\left(1+\alpha^{2}\right) / \gamma B \approx 32 \mathrm{ps}\right.$ in our case). Instead, the whole internal field is relevant for the spin precession, i. e. also contribution from the dipolar field, the exchange and the anisotropy. Since this internal field in non-homogenous 


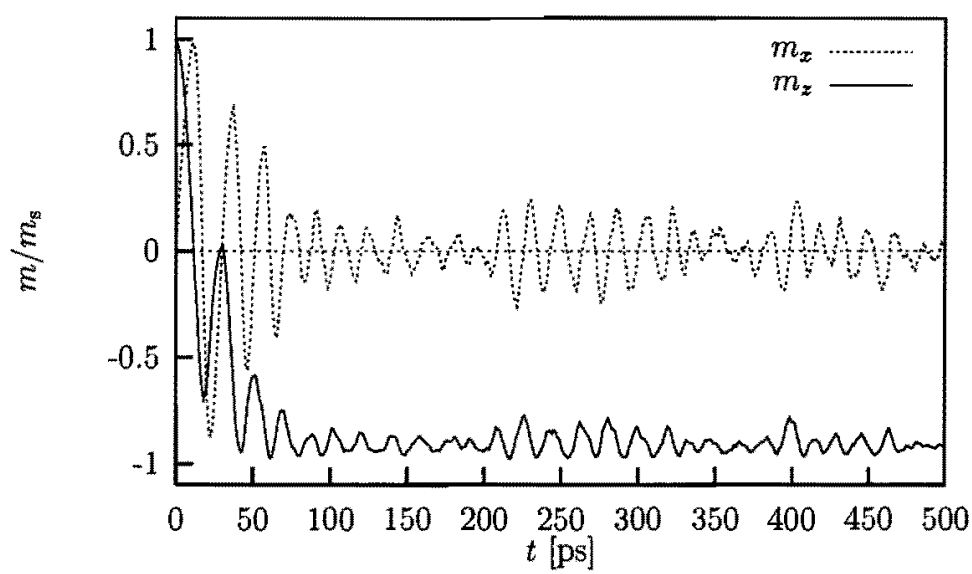

Fig. 1. Reduced magnetization vs. time for a Co system of length $L=4 \mathrm{~nm}$ and diameter $D=2 \mathrm{~nm}$. The data are from Langevin dynamics simulations. $B=1.13$ $\mathrm{T}$ and $k_{\mathrm{B}} T=2.21 \cdot 10^{-23} \mathrm{~J}$

within the system the spins run out of synchronization during time. Due to relaxation effects the system finally reaches its new stable state. Note however, that even after this new stable state is reached the magnetization still keeps on oscillating around the equilibrium value, driven by thermal fluctuations.

\subsection{Thermally Activated Reversal}

On the previous section we discussed fast switching processes where the driving field was above the coercive field. Now we turn to the opposite case, $B<B_{c}$, where the reversal process can only occur when it is thermally activated. We are now interested in the long time and high damping limit $(\alpha=4)$ where the behavior of the particle is governed by thermal fluctuations and where we can use Monte Carlo simulations. Figure 2 shows the typical time dependence of the magnetization of our Co particle. The field $\mathbf{B}$ is set here antiparallel to the initial state, so that the zero-temperature coercive field is given by $B_{c}=2 d_{\mathrm{eff}} V / \mu_{\mathrm{s}}$ which in our case is $B_{c} \approx 1.4 \mathrm{~T}$.

As one can see, the magnetization remains nearly constant for a time period which is rather long as compared to the previous simulation. Then, suddenly, the value of the $z$ component of the magnetization changes its sign. From the large value of the $x$ component of the magnetization at the time of the reversal event one can conclude that the reversal mechanism is mainly a coherent rotation. The value of the switching time is approximately $6.6 \mathrm{~ns}$ in our simulation. However, this thermally activated switching is not a deterministic process as it was the short time dynamics studied before, where the switching followed mainly from the deterministic part of the equation of motion. Instead, the thermal activation process here is a probabilistic event. 


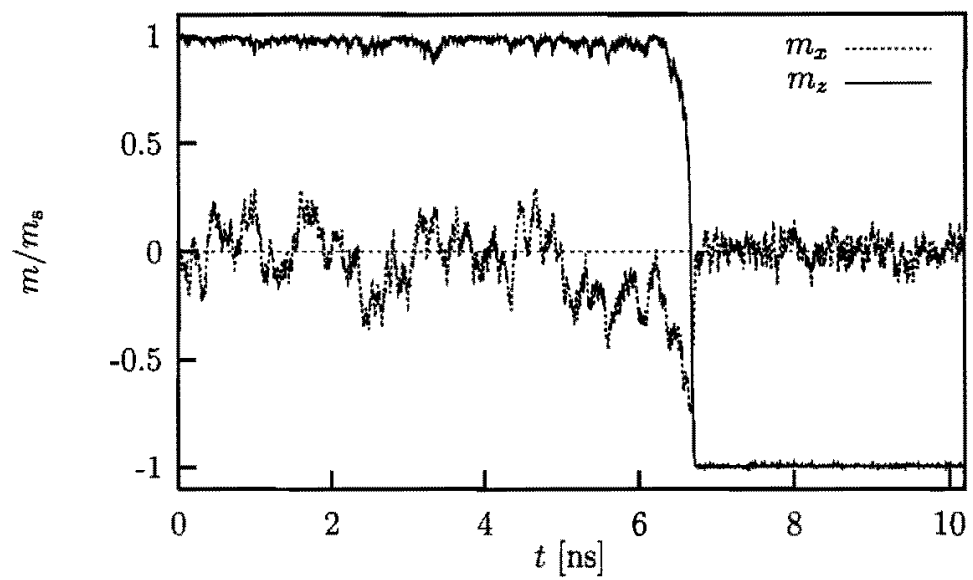

Fig. 2. Reduced magnetization vs. time. The data are from Monte Carlo simulations. $B=1.1 \mathrm{~T}$ and $k_{\mathrm{B}} T=7.1 \cdot 10^{-23} \mathrm{~J}$

The probability distribution $P\left(t_{s}\right)$ for switching events taking place after a time $t_{s}$ is expected to follow an exponential law [9],

$$
P\left(t_{s}\right) \sim \exp \left(-t_{s} / \tau\right)
$$

where $\tau$ is a characteristic time scale. This law is expected to be valid in the range of large time-scales. The characteristic time $\tau$ should follow

$$
\tau=\tau_{0} \exp \left(\Delta E / k_{\mathrm{B}} T\right)
$$

where $\tau_{0}$ is a prefactor and $\Delta E$. an energy barrier which both are related to a certain reversal mechanism (see e. g. $[9,10,26]$ for analytically determined prefactors and energy barriers in different systems and $[5,12,13,24]$ for numerical work on this subject). In general the prefactor may depend on the system parameters, the temperature, the applied magnetic field and the damping constant.

Figure 3 shows a simulated distribution of switching times obtained from 10000 Monte Carlo runs. The distribution shows an exponential behavior and confirms Eq. 6 with an averaged characteristic time of $8.43 \mathrm{ps}$. As one expects, on short time-scales, $t_{s} \ll \tau$, the distribution deviates from the simple exponential behavior since extremely short switching times are less probable.

For the case of a Stoner-Wohlfarth particle with the applied field parallel to the easy axis the energy barrier has the form

$$
\Delta E=d_{\mathrm{eff}} V\left(1-\frac{B}{B_{c}}\right)^{2} .
$$

This energy barrier as well as the prefactor have been calculated by Brown [9] under the assumption that all magnetic moments are parallel, so that the sysm 


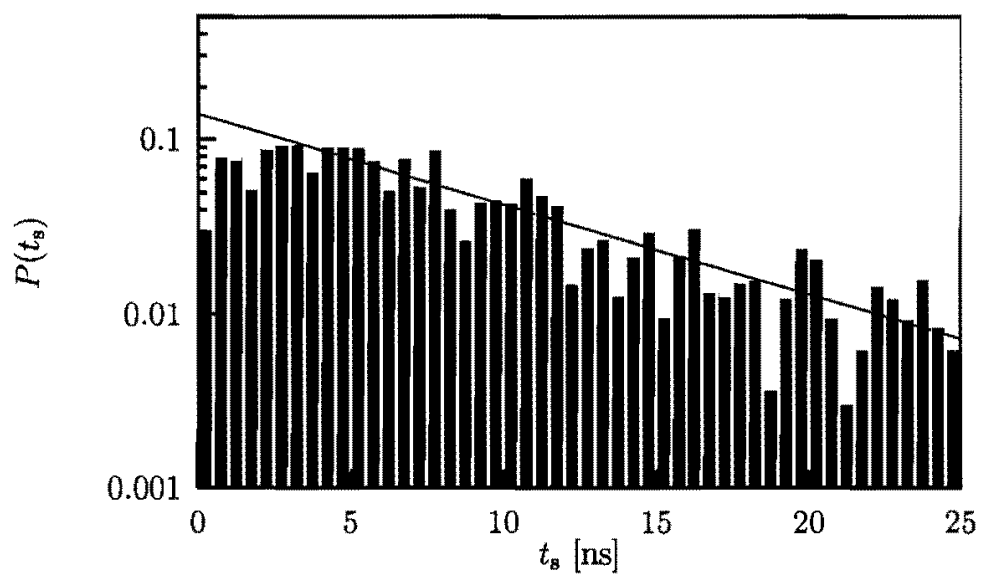

Fig. 3. Distribution $P\left(t_{\mathrm{s}}\right)$ of switching times $t_{\mathrm{s}}$ for our Co particle at $k_{\mathrm{B}} T=$ $7.1 \cdot 10^{-23} \mathrm{~J}$. The data are from 10000 Monte Carlo simulations. The solid line represents the exponential behavior with $\tau=8.43$ ps. $B=1.1 \mathrm{~T}$ as before

tem behaves like one single magnetic moment. In our case, however, the magnetic moments are not strictly parallel so that prefactor and energy barrier are not known offhand.

However, for a further analysis we extract the energy barrier which governs the reversal process from our numerical data. Figure 4 shows the temperature dependence of the characteristic time, i. e. the mean switching time, obtained from our simulations. The slope of the solid line corresponds to the energy barrier for a reversal by coherent rotation with its theoretical value

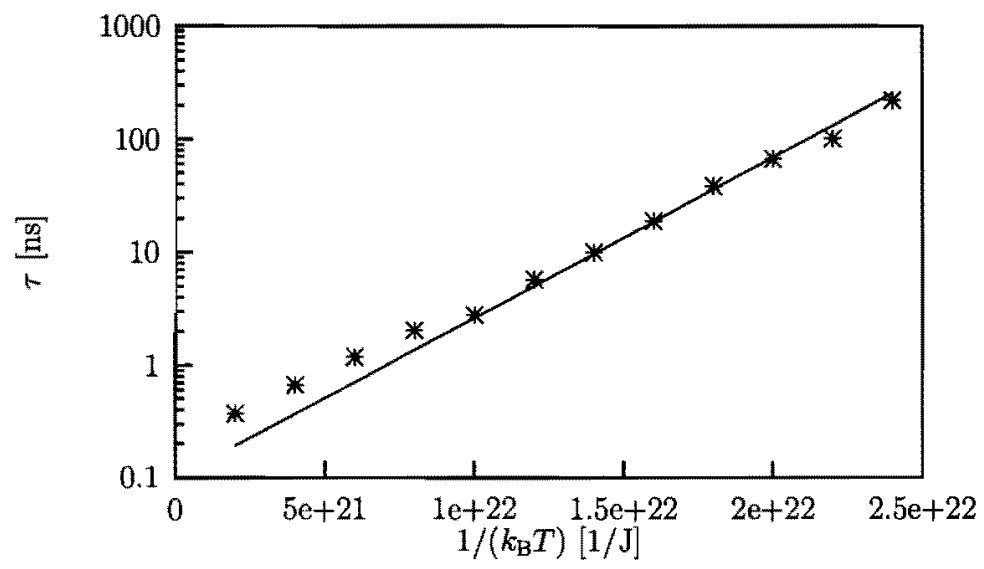

Fig. 4. Characteristic time $\tau$ vs. $1 / k_{\mathrm{B}} T$ for a Co system. The slope of the solid line represents the energy barrier $\Delta E . B=1.1 \mathrm{~T}$ 
$\Delta E=3.27 \cdot 10^{-22} \mathrm{~J}\left(B_{c}=1.39 \mathrm{~T}\right)$ obtained from Eq. 8. Obviously, it is in very good agreement with our numerical data for low enough temperatures.

\section{Conclusions}

In this article, the two basic numerical techniques for the investigation of thermally activated dynamics in magnetic systems were introduced, namely time-quantified Monte Carlo methods and Langevin dynamics simulations.

As an example we investigated magnetization reversal processes in elongated Co nanoparticles. Depending on the strength of the applied field fast switching governed by precession effects can occur or thermally activated switching on a much longer time-scale. We studied two limiting cases, fast processes on a picosecond time scale for higher fields and low damping and slow processes on a time scale of up to one microsecond for lower fields. We discussed the influence of spin precession, the distribution of switching times of the thermal activation process and confirm the predicted exponential behavior. We also found good agreement between the energy barrier deduced from numerical data and theoretical predictions for coherent rotation.

\section{Acknowledgments}

This work was supported by the Deutsche Forschungsgemeinschaft (SFB 491 and project NO290/1) and by the European Union (COST action P3, working group 4).

\section{References}

1. G. Prinz and K. Hathaway: Physics Today 4, 24 (1995)

2. F. J. Himpsel, J. E. Ortega, G. J. Mankey, and R. F. Willis: Adv. Phys 47, 511 (1998)

3. R. W. Chantrell and K. O'Grady in: Applied Magnetism, edited by R. Gerber, C. D. Wright, and G. Asti (Kluwer Academic Publishers, Dordrecht, 1994)

4. A. Lyberatos and R. W. Chantrell: J. Appl. Phys. 73, 6501 (1993)

5. U. Nowak, R. W. Chantrell, and E. C. Kennedy: Phys. Rev. Lett. 84, 163 (2000)

6. C. H. Back, D. Weller, J. Heodmann, D. Mauri, D. Guarisco, E. L. Garwin, and H. C. Siegmann: Phys. Rev. Lett. 81, 3251 (1998)

7. M. Bauer, J. Fassbender, and B. Hillebrands: J. Appl. Phys. 87, 6274 (2000)

8. T. Leineweber and H. Kronmüller, Physica B 275, 5 (2000)

9. W. F. Brown: Phys. Rev. 130, 1677 (1963)

10. H. B. Braun: Phys. Rev. Lett. 71, 3557 (1993)

11. W. Wernsdorfer, E. B. Orozco, K. Hasselbach, A. Benoit, B. Barbara, N. Demoncy, A. Loiseau, H. Pascard, and D. Mailly: Phys. Rev. Lett. 78, 1791 (1997)

12. D. Hinzke and U. Nowak: Phys. Rev. B 61, 6734 (2000) 
13. D. Hinzke and U. Nowak: J. Magn. Magn. Mat. 221, 365 (2000)

14. A. Aharoni: Introduction to the Theory of Ferromagnetism (Oxford University Press, Oxford, 1996)

15. T. Schref, J. Fidler, R. W. Chantrell, and M. Wongsam: Encyclopedia of Materials: Science and Technology (2001), in press

16. A. Hubert and R. Schäfer: Magnetic Domains (Springer-Verlag, Berlin, 1998)

17. S. W. Yuan and H. N. Bertram: IEEE Trans. Mag. 28, 2031 (1992)

18. D. V. Berkov, K. R. Ramstöck, and A. Hubert: Phys. stat. sol. (a) 137, 207 (1993)

19. A. Greiner, W. Strittmatter, and J. Honerkamp: J. Stat. Phys. 51, 95 (1988)

20. J. L. García-Palacios and F. J. Lázaro: Phys. Rev. B 58, 14937 (1998)

21. K. Binder and D. W. Heermann in: Monte Carlo Simulation in Statistical Physics, edited by P. Fulde (Springer-Verlag, Berlin, 1997)

22. F. Reif: Fundamentals of statistical and thermal physics (McGraw-Hill Book Company, New York, 1967)

23. R. Smirnov-Rueda, O. Chubykalo, U. Nowak, R. W. Chantrell, and J. M. Gonzáles: J. Appl. Phys. 87, 4798 (2000)

24. U. Nowak in: Annual Reviews of Computational Physics IX, edited by D. Stauffer (World Scientific, Singapore, 2000), p. 105

25. E. C. Stoner and E. P. Wohlfarth: Philos. Trans. R. Soc. London Ser. A 240, $599(1949)$

26. W. T. Coffey, D. S. F. Crothers, J. L. Dorman, Y. P. Kalmykov, E. C. Kennedy, and W. Wernsdorfer: Phys. Rev. Lett. 80, 5655 (1998) 